\title{
Prospects for the high-luminosity LHC
}

\author{
S. Jezequel ${ }^{1}$ on behalf of the ATLAS and CMS Collaborations
}

${ }^{1}$ LAPP IN2P3-CNRS and Université de Savoie, 9 Chemin de Bellevue F-74940 Annecy-Le-Vieux, France

\begin{abstract}
This note reviews the main physics topics accessible with the high-luminosity LHC program (HL-LHC). It should deliver p-p collisions at $\sqrt{s}=14 \mathrm{TeV}$ with an integrated luminosity of $3000 \mathrm{fb}^{-1}$. Results are presented in perspective with the previous period with ten times less luminosity. The ATLAS and CMS collaborations released expected results for this program assuming similar detector performance as today within more difficult conditions. The Higgs boson branching ratios and couplings to fermions/bosons will be measured at few percent level. The main discovery limits for the search of new particles or phenomena beyond the Standard Model are presented.
\end{abstract}

Keywords: LHC, High-Luminosity, ATLAS, CMS

\section{Introduction}

The Large Hadron Collider (LHC) [1] has delivered proton-proton collisions over the period 2009-2012. Most of the data have been produced at center-of-mass energies $(\sqrt{s})$ equal to 7 and $8 \mathrm{TeV}$ for a total luminosity of $29 \mathrm{fb}^{-1}$, out of which $\sim 25 \mathrm{fb}^{-1}$ are usable for physics analysis by the ATLAS [2] and CMS [3] collaborations. The main physics outcome was the discovery of a Higgs boson at a mass of $125 \mathrm{GeV}$ [4] [5] which is an important ingredient for the understanding of the electroweak symmetry breaking mechanism. The current results (branching ratios, couplings, ...) are compatible with the Standard Model (SM) expectations. In addition, many limits have been set on the search of new particles beyond the Standard Model including supersymmetry and exotic models. This period, called Run 1 , is used as a reference for detector performance.

The LHC is currently stopped for accelerator upgrade and should restart beginning 2015 for a period of three years. An integrated luminosity of $75-100 \mathrm{fb}^{-1}$ at a center of mass energy from 13 to $14 \mathrm{TeV}$ (Run 2) is expected. Afterwards, two data taking periods called LHC (approved) and HL-LHC (not approved yet) are expected to start in $\sim 2019$ and $\sim 2023$. The two periods should deliver $300-350 \mathrm{fb}^{-1}$ (incorporating the Run
2 data) and $3000 \mathrm{fb}^{-1}$ at $\sqrt{s}=14 \mathrm{TeV}$. During the two shutdowns prior to these periods, the two experiments will upgrade their detectors [6] [7] [8] to keep similar detector performance as Run 1 but under more severe conditions (more radiation, more pileup, higher trigger rate,...). Based on the current experience with data analysis at Run 1 and the expected detector performance during the 300 and $3000 \mathrm{fb}^{-1}$ campaigns, the ATLAS and CMS collaborations have recently provided expected precisions on the measurements of the Higgs boson properties and the accessible parameters for the search of new physics beyond the Standard Model (BSM). The CMS collaboration also included studies in view of a High-Energy LHC program (HELHC) whose target is to deliver an integrated luminosity of $300 \mathrm{fb}^{-1}$ at $\sqrt{s}=33 \mathrm{TeV}$. More recent studies [9] [10] were released just after the presentation.

\section{Higgs boson properties}

The precise measurement of the Higgs boson properties is very important. The key properties are the mass, the spin-parity and the couplings to the different fermions and bosons. The first two items will be addressed with Run 2 data. 
With the high statistics collected during the $300 \mathrm{fb}^{-1}$ campaign, the different Higgs-decay branching ratios will be measured with a precision of $10-15 \%$. At this level, the current theory uncertainties cannot be neglected anymore. Assuming the same experimental systematic uncertainty, the theoretical uncertainty is dominant for diboson final state. The exptected precision on the signal strength $\mu$ (cross section times branching ratio normalised to the SM Higgs prediction) is displayed in Figure 1 for different Higgs decay channels.

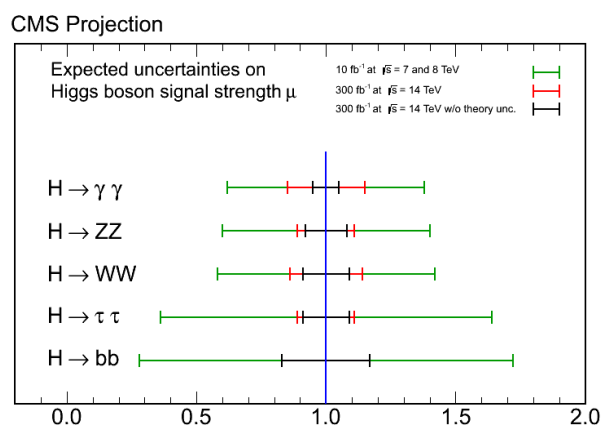

Figure 1: Estimated precision of the signal strength determination for a SM Higgs boson with $10 \mathrm{fb}^{-1}$ at 7-8 TeV (green), $300 \mathrm{fb}^{-1}$ at $14 \mathrm{TeV}$ with current systematic uncertainty (red) or removing the theoretical contribution (black) [11].

With $3000 \mathrm{fb}^{-1}$, very rare Higgs decay channels become accessible. One example is $\mathrm{H} \rightarrow \mu \mu$. The signalto-background ratio for this inclusive channel is only $0.2 \%$ but the expected narrow peak in the $\mu \mu$ invariant mass allows a signal extraction, resulting in an expected signal significance larger than $6 \sigma$. The $\mu \mu$ invariant mass plot (Figure 2) shows the expected signal compared to the large continuous Drell-Yan background.

The Higgs coupling to fermions and bosons is measured from the Higgs decay branching ratios but also from Vector Boson Fusion (VBF) and associated production $(\mathrm{WH} / \mathrm{ZH}$ and $t \overline{\mathrm{H}})$. The available results on associated production are currently restricted to $\mathrm{H} \rightarrow$ $\gamma \gamma$ decay channel with $\mathrm{W} / \mathrm{Z}$ decaying in leptons and top into lepton+jet decay. With $3000 \mathrm{fb}^{-1}$, the Signal/Background ratio is expected to be $20 \%$ for $t \vec{t} \mathrm{H}$, $10 \%$ for $\mathrm{ZH}$ and $2 \%$ for $\mathrm{WH}$.

Table 1 summarizes the ATLAS expectations [12] with 300 and $3000 \mathrm{fb}^{-1}$ on the precision of couplings in a minimal coupling fit (only two independant scale factors $K_{V}$ and $K_{F}$ ). All the Higgs channels are included in the fit. The systematic uncertainties include the current theoretical ones.

CMS made a similar analysis [11] addressing a sce-

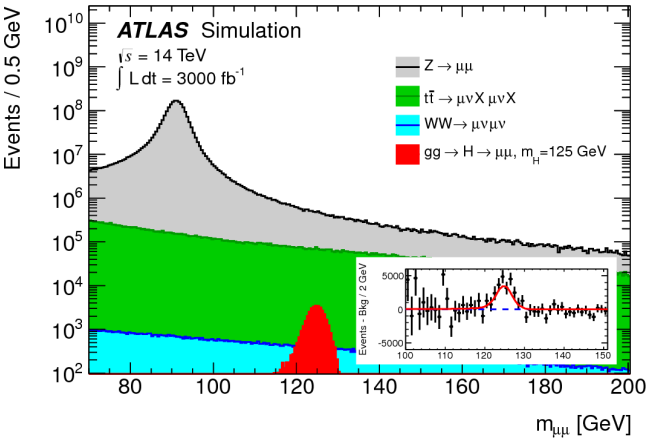

Figure 2: Expected invariant mass distribution for the inclusive $\mathrm{H} \rightarrow$ $\mu \mu$ channel with $3000 \mathrm{fb}^{-1}$. The inset shows the expectation for the signal after the substraction of the fitted background [7].

\begin{tabular}{ccc}
\hline & $300 \mathrm{fb}^{-1}$ & $3000 \mathrm{fb}^{-1}$ \\
\hline$K_{V}$ & $3.0 \%(5.6 \%)$ & $1.9 \%(4.5 \%)$ \\
$K_{F}$ & $8.9 \%(10 \%)$ & $3.6 \%(5.9 \%)$
\end{tabular}

Table 1: Expected precision for the determination of the coupling scale factors $K_{V}$ and $K_{F}$ with 300 and $3000 \mathrm{fb}^{-1}$. Numbers in brackets include current theory systematic uncertainties [12].

nario similar to the ATLAS one and an ultimate one where the theoretical uncertainty is divided by a factor two and the experimental one scaled by $1 / \sqrt{\mathcal{L}}$ with $\mathcal{L}$ defined as the integrated luminosity. The last case should be considered as a target for theorists and experimentalists and provides a few percent precision.

The Higgs trilinear coupling measurement is also an important subject for the High-Luminosity program and is achieved through the selection of double Higgs final state. Previous studies have shown that the measurement of the double Higgs production is possible with $3000 \mathrm{fb}^{-1}$. Promising final states are $\mathrm{H} \rightarrow b \bar{b} \gamma \gamma$ where one of the Higgs can be precisely reconstructed to reduce background.

\section{Searches beyond Standard Model}

\subsection{Vector Boson scattering}

A major reason for the existence of new particles or interactions at the $\mathrm{TeV}$ energy scale has been the prediction of diverging cross section for vector boson scattering in the longitudinal mode which would violate unitarity at this scale. In the SM, the Higgs boson controls this divergence. To probe BSM physics in an extended Higgs sector, ATLAS [13] presents an analysis for three final states based on high-mass diboson resonances associated with two forward jets: WW into lepton + jet, 
WW in the dilepton channel and ZZ into 4 leptons. For the lepton+jet WW final state, the 95\% CL exclusion limit for the $a_{4}$ parameter in the generalised electroweak chiral Lagrangian is $0.066 / 0.016$ with $300 / 3000 \mathrm{fb}^{-1}$. For different reference values of resonance masses and couplings in the ZZ final state, the discovery at $5 \sigma$ can be done only with $3000 \mathrm{fb}^{-1}$.

\subsection{Supersymmetry}

The observation of the Higgs boson with a mass of $125 \mathrm{GeV}$ increases the need of finding a solution to the stability of its mass to large quantum corrections (the so-called hierarchy problem). Natural supersymmetry is one of the most effective and popular solutions to this problem. No evidence of this model has been found yet.

The ATLAS [14] and CMS [11] collaborations searched for three particle families : generic squarks and gluinos, light top squarks (stop) and SUSY partners of the electroweak gauge bosons (EWKinos). Assuming a mass equal to zero for the Lighest Supersymmetric Particle, the current $95 \%$ exclusion limits with Run 1 data are respectively $\sim 1.8 \mathrm{TeV}$ for $\tilde{g}$ in a simplified squark-guino model (assuming $\mathrm{m}_{\tilde{g}}=\mathrm{m}_{\tilde{q}}$ ), $\sim 40$ and $\sim 640 \mathrm{GeV}$ for the top squark depending on the decay channel and $320 \mathrm{GeV}$ EWKinos. All the topologies of these events include large missing transverse energy (MET) and different numbers of leptons and jets. The expected discovery and exclusion limits with 300 and $3000 \mathrm{fb}^{-1}$ in the plane $\mathrm{m}_{\tilde{g}}-\mathrm{m}_{\tilde{q}}$ are displayed in Figure 3.

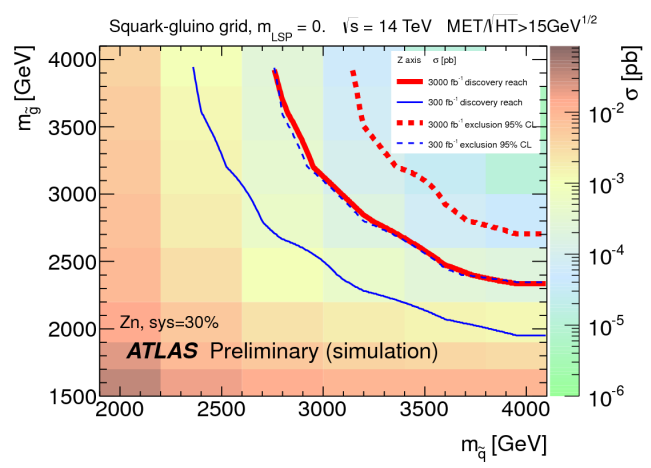

Figure 3: The 95\% CL exclusion limit (solid lines) and $5 \sigma$ discovery reach (dashed lines) in a simplified squark-gluino model with massless neutralino with $300 \mathrm{fb}^{-1}$ (blue lines) and $3000 \mathrm{fb}^{-1}$ (red lines) [7].

Figure 4 summarizes the exclusion limits computed by CMS for the three particle types with $300 \mathrm{fb}^{-1}$, for the HL-LHC and the HE-LHC programs. Since the top squarks and EWKinos can be excluded up to $1 \mathrm{TeV}$ or more, the High-Luminosity LHC program gives the opportunity to confirm or fully reject the natural supersymmetry.

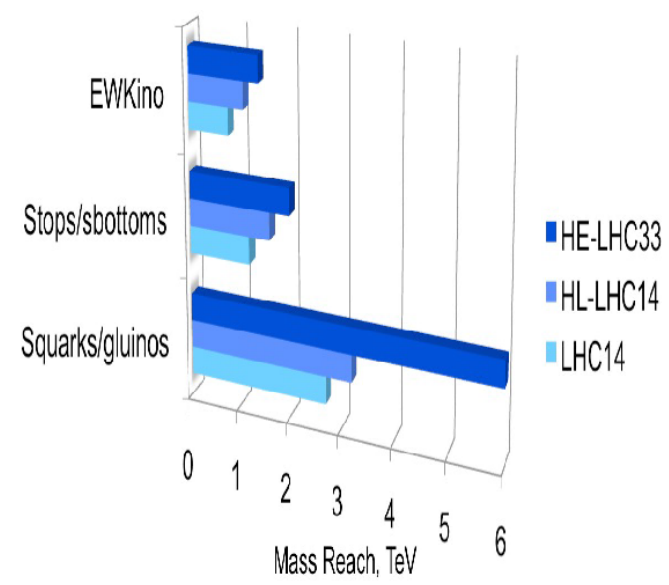

Figure 4: Reach of searches for supersymmetry at CMS with $300 \mathrm{fb}^{-1}$ (LHC14), the HL-LHC (HL-LHC14) and HE-LHC (HE-LHC33) programs [11].

\subsection{Exotics}

Excluding natural supersymmetry makes even more important to search for alternative solutions of the hierarchy problem like exotic particles. The search for exotic physics can be done through high-mass resonances. ATLAS [15] and CMS [11] performed the studies for two different final states : dileptons and $t \bar{t}$ pairs.

The dilepton final state (ee or $\mu \mu$ ) is one of the most simple and robust signatures of a new particle. The $t \bar{t}$ final state is a probe for the search of Kaluza-Klein gluon $g_{K K}$ and Z' boson in the Topcolour model. The top pair is selected through lepton+jets or dilepton final state.

Table 2 shows the different expected limits for the different models with 300 and $3000 \mathrm{fb}^{-1}$. The gain in integrated luminosity by a factor 10 raises the sensitivity to high-mass resonances by up to $2.4 \mathrm{TeV}$ for $t \bar{t}$ resonances and $1.3 \mathrm{TeV}$ for the dilepton $\mathrm{Z}_{S S M}{ }_{S}$ resonance.

\begin{tabular}{ccc}
\hline model & $300 \mathrm{fb}^{-1}$ & $3 \mathrm{ab}^{-1}$ \\
\hline$g_{K K}$ & 4.3 & 6.7 \\
$\mathrm{Z}_{\text {Topcolour }}$ & 3.3 & 5.5 \\
\hline $\mathrm{Z}^{\prime}{ }_{S S M} \rightarrow e e$ & 6.5 & 7.8 \\
$\mathrm{Z}^{\prime}{ }_{S S M} \rightarrow \mu \mu$ & 6.4 & 7.6 \\
\hline
\end{tabular}

Table 2: Summary of the expected limits for different exotics models for pp collisions at $\sqrt{s}=14 \mathrm{TeV}$. All limits are quoted in TeV [7]. 
Some BSM models predict the existence of leptoquarks (LQ) that carry both baryon and lepton numbers and couple to both quarks and leptons. CMS [11] computed the expected search limits assuming that leptoquark decays only in electron + jet. The final state is 2 electrons +2 jets. Two assumptions are made on the Signal/Background ratios :

- $\mathrm{S} / \mathrm{B}<1$ : Working point in the Run 1 range (conservative)

- $\mathrm{S} / \mathrm{B} \sim 1$ : Working points at HL-LHC

The results are summarised in Table 3 and can be compared to the current limits of $0.8 \mathrm{TeV}$.

\begin{tabular}{cccc}
\hline Scenario & $300 \mathrm{fb}^{-1}$ & $3000 \mathrm{fb}^{-1}$ & HE-LHC \\
\hline $\mathrm{S} / \mathrm{B}<1$ & 1.6 & 1.8 & 2.5 \\
$\mathrm{~S} / \mathrm{B} \sim 1$ & 1.7 & 2.3 & 3.5 \\
\hline
\end{tabular}

Table 3: Mass reach $(\mathrm{TeV})$ of the lepto-quark search in the eejj channel for two different values of the analysis working points [11]

\subsection{Flavour Changing Neutral Current in top decays}

The HL-LHC will provide a very large sample of topquark decays which can be used to gain orders of magnitude for the search of Flavour Changing Neutral Current (FCNC) in top decays. The FCNC top-quark decay $(\mathrm{t} \rightarrow \mathrm{Zq}$ and $\mathrm{t} \rightarrow \gamma \mathrm{q}, \mathrm{q}=u, c)$ is forbidden at tree level in the SM but is enabled through loop diagrams at values smaller than $10^{-12}$. There are several Standard Model extensions which enhance this branching ratios up to $10^{-5}-10^{-4}$.

The current limits at $95 \% \mathrm{CL}$ on these decays channels are of the order of $10^{-2}$ for $\mathrm{q} \gamma$ and $10^{-3}$ for qZ (Figure 5). ATLAS [16] and CMS [11] computed the expected exclusion limits using $t \bar{t}$ events with 300 and $3000 \mathrm{fb}^{-1}$ data. The $t \bar{t}$ pair is tagged with one top decaying into bW. FCNC is tested on the other top, looking for $\mathrm{qZ}$ or $\mathrm{q} \gamma$ final state. The expected 95\% CL exclusion limits with 300 and $3000 \mathrm{fb}^{-1}$ are of the order of few $10^{-5}-10^{-4}$ and displayed in Figure 5 .

\section{Conclusion}

The high luminosity LHC program will measure at the percent level the Higgs couplings to fermions and bosons. Many BSM models could be confirmed or fully excluded. For some other models, the phase space coverage will be significantly extended. The presented analyses are based on the Run 1 experience and detector

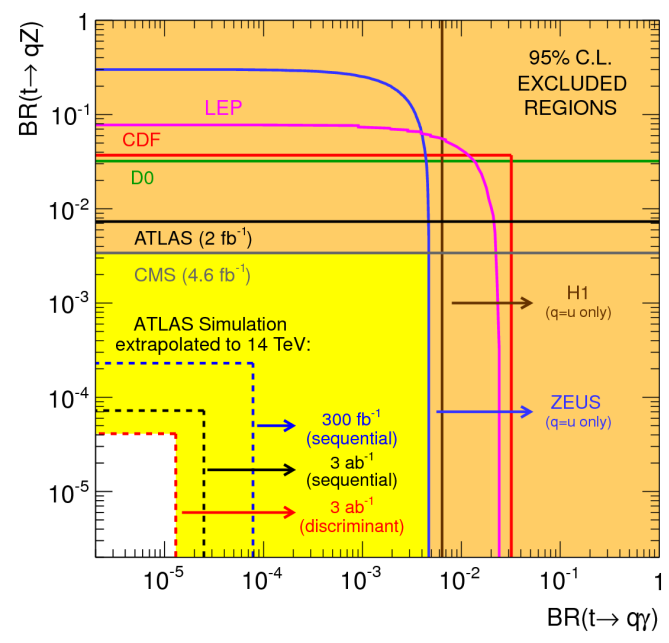

Figure 5: The present and expected 95\% CL observed limits on $\mathrm{BR}(\mathrm{t} \rightarrow \gamma \mathrm{q})$ versus $\mathrm{BR}(\mathrm{t} \rightarrow \mathrm{qZ})$ plane [7].

performance modelling. Such studies will continue in order to improve the detector response description and to introduce new physic channels.

\section{References}

[1] L. Evans and P. Bryant, JINST 3 (2008) S08001

[2] The ATLAS Collaboration, JINST 3 S08003 (2008).

[3] The CMS Collaboration, JINST 08033 (2008).

[4] The ATLAS Collaboration, Phys. Lett. B716 (2012) 1, arXiv:1207.7214 [hep-ex].

[5] The CMS Collaboration, Phys. Lett. B 716 (2012) 30, arXiv:1207.7235 [hep-ex].

[6] The ATLAS Collaboration, CERN-LHCC-2011-12, http://cds.cern.ch/record/1402470.

[7] The ATLAS Collaboration, CERN-LHCC-2012-22, http://cds.cern.ch/record/1502664.

[8] The CMS Collaboration, http://cds.cern.ch/record/1355706.

[9] The CMS Collaboration, http://cds.cern.ch/record/1565454.

CERN-LHCC-2011-006,

CMS-NOTE-2013-002,

[10] The ATLAS COllaboration, ATL-PHYS-PUB-2013-006, http://cdsweb.cern.ch/record/1558703.

[11] The CMS Collaboration, CMS NOTE-2012/006, http://cds.cern.ch/record/1494600.

[12] The ATLAS Collaboration, ATLAS-PHYS-PUB-2012-004, http://cdsweb.cern.ch/record/1484890.

[13] The ATLAS Collaboration, ATLAS-PHYS-PUB-2012-005, http://cdsweb.cern.ch/record/1496527.

[14] The ATLAS Collaboration, ATLAS-PHYS-PUB-2013-002, http://cdsweb.cern.ch/record/1512933.

[15] The ATLAS Collaboration, ATLAS-PHYS-PUB-2013-003, http://cdsweb.cern.ch/record/1516108.

[16] The ATLAS Collaboration, ATLAS-PHYS-PUB-2012-001, http://cds.cern.ch/record/1472518. 